\title{
Analysis and Validation of AUTOSAR Models
}

\author{
Julian Kienberger ${ }^{1}$, Pascal Minnerup ${ }^{2}$, Stefan Kuntz ${ }^{3}$ and Bernhard Bauer ${ }^{1}$ \\ ${ }^{1}$ Department of Computer Science, University of Augsburg, Augsburg, Germany \\ ${ }^{2}$ fortiss GmbH, An-Institut Technische Universität München, Munich, Germany \\ ${ }^{3}$ Division Powertrain, Continental Automotive GmbH, Regensburg, Germany
}

Keywords: Data Dependency Analysis, AUTOSAR, Model-Driven Development, Multi-core, Parallelization.

\begin{abstract}
As the rise of single-core processing power is exhausted due to technical limitations, the automotive branch is forced to migrate its control unit software to architectures that feature multiple Independent Execution Units (IEUs). This policy shift brings along new problems resulting from the tremendously increased complexity of such systems. Facing these challenges, software engineers have to cope with possible data inconsistencies caused by, e.g., race conditions or cycles. Being an important and standardized software architecture for electronic control units, the Automotive Open System Architecture (AUTOSAR) provides the basis for tools that support the complexity handling when migrating to architectures with multiple IEUs. Our concept is realized by a tool that executes data dependency analyses directly on AUTOSAR models, determines critical dependencies, automatically solves trivial problems and provides semi-automatic resolution of advanced conflicts. To support the actual parallelization of the system, the tool additionally determines groups of executable units that are suitable to run on a common IEU. This appreciably facilitates the validation of AUTOSAR models and the search for a good mapping of the processing tasks to IEUs.
\end{abstract}

\section{INTRODUCTION}

In recent years, it has become apparent that the performance of single core processors is almost completely exhausted, because they are approaching their limits concerning clock speed and memory speed (Gleim and Schüle, 2012). By all indications, the further rising of the clock speed is unreasonable from an economical and technical point of view, because it inevitably leads to a disproportionate growth of the CPU's power consumption and an enormous rise of the corresponding heat dissipation efforts (Sutter, 2005).

Nevertheless, car manufacturers and the associated supplying industry are aiming at adding further functionality to Electronic Control Units (ECUs) which increases complexity as well as required processing performance (Deubzer et al., 2010; Schäuffele and Zurawka, 2010). Moreover, they intend to decrease the number of ECUs in order to save space and reduce weight. These intentions strengthen the endeavor to find a possibility for ramping up the available computing power.

The development of embedded architectures that feature multiple IEUs is a promising approach to meet these recent challenges and is hence becoming increasingly important (Wirbel, 2011). Therefore, the companies of the automotive branch are migrating to such architectures, which are often simply referred to as "multi-core" architectures, although this is just one specific solution that is frequently used to vicariously represent the whole idea of parallel computing (Bohn et al., 2011).

The distribution of the functional blocks, i.e. atomic computational tasks, to several IEUs solves the aforementioned problems with power consumption, heat dissipation and processing performance. However, it is eminently augmenting the complexity of software due to dependencies between separately processed but still interdependent data elements including problems like race conditions, dead locks, non-determinism and insufficient load balancing (Padberg and Denninger, 2013; Patterson, 2010). Therefore new methods and tools are needed that assist software engineers in designing and implementing such systems (Multicore, 2011; Shih et al., 2009; Eißenlöffel, 2012).

One approach is AUTOSAR, which is a standardized platform developed by several leading manufacturers and suppliers of the automotive sector.

274 Kienberger J., Minnerup P., Kuntz S. and Bauer B. Analysis and Validation of AUTOSAR Models. DOI: $10.5220 / 0004701002740281$

In Proceedings of the 2nd International Conference on Model-Driven Engineering and Software Development (MODELSWARD-2014), pages 274-281 ISBN: 978-989-758-007-9

Copyright (C) 2014 SCITEPRESS (Science and Technology Publications, Lda.) 
AUTOSAR specifies a uniform software architecture and defines interfaces for communication as well as configuration formats which facilitate the exchange of ECU software, assure its possible reuse and make it scalable (AUTOSAR, 2013).

Hence, there is a strong demand for tools that both harness the advantages of AUTOSAR and support the complexity handling when migrating to architectures featuring multiple IEUs (Sodan et al., 2010; Gehrke et al., 2006). The latter is mainly about conflicts within specific execution sequences that can be avoided by imposing constraints on the involved functional blocks. Conflicts can be, e.g., data not being available in time or data being read inconsistently.

Facing these problems, our research endeavors led to the following concept:

- determination of the atomic execution units' data dependencies and their types by means of a static data dependency analysis performed directly on AUTOSAR models (Section 3)

- automatic imposition of mandatory constraints and deduction of existing conflicts (Subsection 3.2)

- conflict detection and validation through provision of semi-automatic resolution actions (Subsection 3.3)

- determination and storage of virtually isolated functional block regions to effectively support the subsequent search for a proper "task to IEU" mapping when trying to parallelize a system (Section 4).

These aspects are framed by a description of the preconditions and challenges (Section 2) and a case study that illustrates the utilization of the tool (Section 5).

\section{PRECONDITIONS AND CHALLENGES}

In order to properly understand the context of a data dependency analysis involving execution orders, it is crucial to be aware of the initial situation. Generally speaking, there are two basic starting points:

- having a data dependency graph (without timing constraints imposed) and trying to specify a proper execution order

- having already given an execution order and analyzing the "cycle problems" of the corresponding data dependency graph

The former description corresponds to our use case. The foundation of the analysis is a structural model that does not contain any constraints. It merely consists of AUTOSAR's Software Components (SW-Cs) together with their Internal Behavior including one or more Runnable Entities (REs), which are the smallest (and therefore the atomic) executable entities in the scope of AUTOSAR. A sketch of such a model is presented in Figure 1.

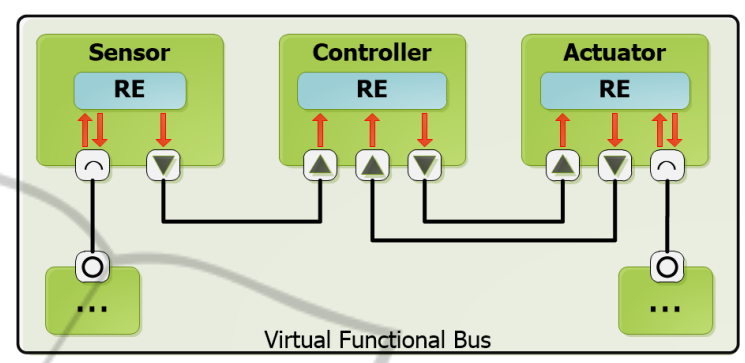

Figure 1: This simplified version of a typical sensor-actor system is an example of a structural AUTOSAR model without timing constraints. The arrows and the small blackand-white boxes indicate the communication direction between the REs.

The Internal Behavior comprises the communication taking place within an SW-C (between several of its REs) and between different SW-Cs. Since there can be multiple instances of one single RE, it is important to mention that each instance is actually independent and has its own data dependencies.

Such a dependency arises from the interaction between at least two Runnable Entity instances (REIs). As each REI can read and write variables, a data dependency between two REIs exists if, e.g., one of them reads a variable that has been written by the other one. Exceeding this trivial case, multiple $\mathrm{read} /$ write actions can form a direct circular dependency between two REIs or a transitive dependency between multiple ones (a cycle). Figure 2 shows an example conflict and its possible solution.

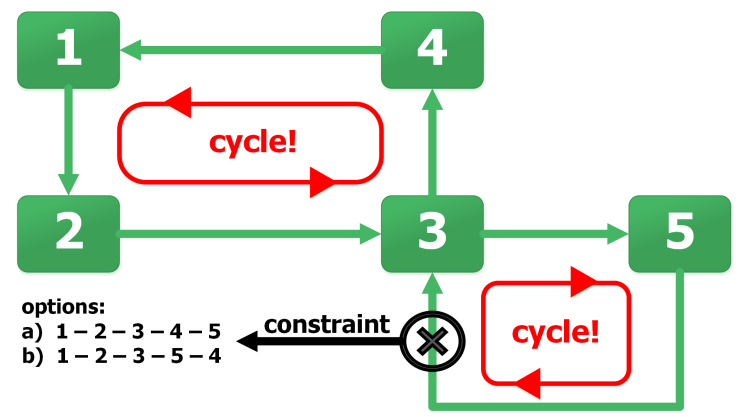

Figure 2: This figure shows a conflict example where the calculated output from "REI 3" is a necessary input for the calculation of "REI 5" and vice versa. This cycle has to be broken up via a constraint that allows the transfered data to be as old as one computing cycle or that imposes an execution order on the involved REIs (see Section 3.2). 
Cycles are particularly important as automotive control software mainly runs periodic tasks, and it immediately suggests itself to look upon the included REIs from a temporal perspective. In this context, the commonly used unit is "computing cycle", which we define as the time elapsed between two events that involve periodically activated tasks being guided by the slowest (least triggered) task occurring.

In a single-core system, the execution order of the REIs has been carefully designed and tested according to the knowledge about triggering frequencies and computing cycles. Changing the execution order can lead to an REI reading invalid data because every data element has to be produced before it can be consumed. However, such a rigid execution order cannot be maintained when migrating to, e.g., a multi-core system, because there is a need for more freedom concerning the execution order of the REIs that may run on different IEUs. Sticking to the heavily constrained order of a single-core system would result in using only one IEU. For a software engineer, it is a tightrope walk to preserve as much freedom as possible and simultaneously prevent the system from entering problematic states that cause, e.g., race conditions, data inconsistencies or dead locks. Moreover, too many constraints might impede achieving a proper load balancing (equal IEU workloads) and therefore the speedup, which is usually a major goal of migration, can be appreciably restrained.

Facing the significant rise of the systems' complexity, it becomes clear that discovering possible conflicts by use of data dependency analyses is vital when several IEUs shall be used.

\section{MODEL ANALYSIS AND VALIDATION}

Our concept is based on a static data dependency analyses performed on AUTOSAR models. Its goals are to discover design weaknesses, to automatically solve trivial pitfalls, to support the elimination of the remaining conflicts (mainly cycles) and to write back the modifications to the model. In detail, the concept is to analyze the connections (data dependencies) between the Executable Entities (i.e. the REIs) contained in the SW-Cs and possible execution sequences of them. By classifying the detected data dependencies, it is feasible to restrict all possible execution sequences to a set that provides every REI with its required input data in time, which ensures that consistency constraints are met.

\subsection{Development and Scope}

The implementation uses the Model Analysis Framework (MAF), which is based on the Eclipse Modeling Framework and provides "a core framework [...] allowing the implementation of dynamic model analysis" (Saad, 2009; Eclipse Foundation, 2009). Its main application (and our use case) is the execution of data-flow analyses as described in (Saad and Bauer, 2013). Figure 3 shows MAF together with parts of the Eclipse tool stack.

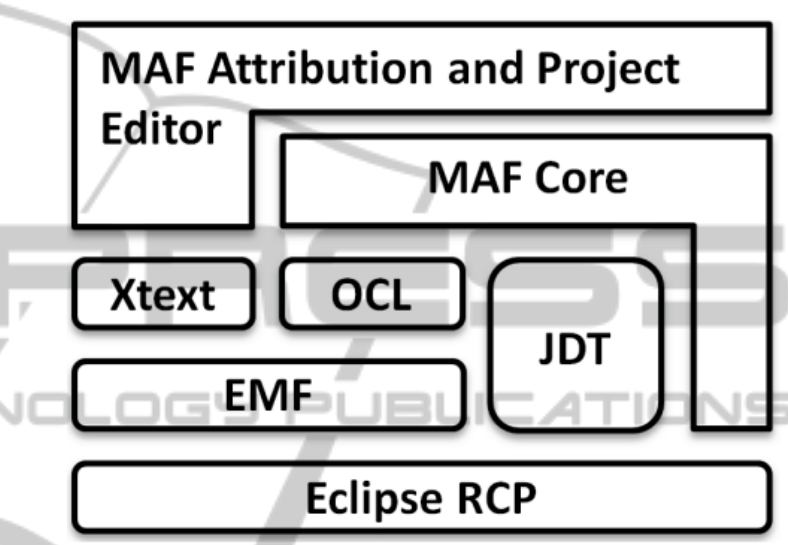

Figure 3: This figure illustrates the collaboration of MAF with established frameworks and platforms for Eclipse as well as with UML's Object Constraint Language (OCL).

The implementation is realized as a plug-in for the AUTOSAR Tool Platform (Artop), which serves as Eclipse infrastructure for the construction of AUTOSAR tools (Artop Group, 2012). It virtually acts as "persistence layer" enabling common base functionality like easy access on AUTOSAR models that adhere to specific AUTOSAR meta-model versions. Its major components include features like meta model implementations, model comparison, model validation, explorers, editors and a code generation infrastructure.

For the processing, it is necessary to analyze the specified Internal Behavior of the REIs. As SW-Cs exchange data via so-called Assembly Connectors, their connection points to a software component are referred to as Ports. Port interfaces specify, e.g., variables, operations, triggers and mode switches of a port when using the connectors. Regarding communication within SW-Cs, the data sent from an REI and received by another one is represented as VariableAccess (VA) connected to them. In general, variables (as part of a port or local variables of a component) play a significant role for the later analysis. There are several kinds of VAs: 
- outgoing VAs:

- dataSendPoint

- dataWriteAccess

- writtenLocalVariable

- incoming VAs:

- dataReceivePointByValue

- dataReceivePointByArgument

- dataReadAccess

- readLocalVariable

The analysis concept covers all listed elements and also includes data dependencies that arise from interrunnable communications of REIs in one (atomic) SW-C.

\subsection{Analysis Steps}

The aforementioned MAF is used in the first phase of the analysis. Here, the intermediate goal is to properly "prepare" the succeeding search of the model for potentially problematic data dependencies. This is done by annotating the AUTOSAR model and storing useful information in specific hash maps that support the efficient execution of the subsequent analysis. To be exact, the model is traversed and relationships like predecessors/successors are stored in "quick access maps" (storage maps within MAF's global object container) in order to reduce the effort that is required to navigate highly complex AUTOSAR models during the analysis. In other words, the data flow analysis is released from carrying out the same "expensive" traversal steps and calculations several times by computing required links once before the actual analysis happens. This approach does tremendously speed up the following search for dependencies. The created quick access maps also enforce that only one dataflow analysis result occurs for each object although one single RE may be referenced several times in different contexts (RE instances).

Based on the data collected in this "pre-analysis", it is now possible to utilize the precomputed access maps for quickly iterating over all instances of the Runnable Entity that is currently being processed. An instance can be referred to using the corresponding RE in the AUTOSAR model together with the path of the specific instantiation. In AUTOSAR, this path is stored as a list of SwComponentPrototypes in the ComponentInCompositionInstanceRef. Using this path, the analysis can cope with the multiple instantiations.

For finding the partner of data exchanges, two different cases have to be considered: the writing REI can either access a variable within the SW-C (local variables and inter-runnable variables) or outside the
SW-C via a port. If it accesses a local variable, the consuming REI is the entity reading from the same local variable. The only thing to note is that the reading entity and the writing entity have to share the same instantiation path as described above. This makes sure that the REIs of the specific SwComponentPrototypes are connected. If the writing REI accesses a port, the connections of it have to be considered. It can be connected to another port of a different component using an AssemblySwConnector. The port receiving the data can be accessed by an RE. The correct instance of this RE is the consumer of the data dependency. Alternatively the data can be delegated to a port of an inner component using a DelegationConnectorPrototype. In addition to these data dependencies, AUTOSAR of course enables the modeling of function calls that bring along data- and control-flow dependencies.

Having found all data dependencies of the occurring REIs, the next step is to set the execution of them into an temporal perspective by determining whether the dependencies act as Forward Dependency (FD) or Backward Dependency (BD) with regards to their execution order. FDs and BDs can be characterized as follows:

- Forward Dependencies are marked by the fact that in one computing cycle, the variables are first written and afterwards read. In detail an "initial REI" writes to a variable that is thereafter read by a "target REI" which is a successor according to the quick access maps created within the preanalysis.

- Backward Dependencies are all dependencies remaining if FDs are not taken into consideration. This means that a variable is first read and afterwards written in the scope of one computing cycle. Considering different computing cycles, the very last access to a variable (before the computing cycle ends) is a write access. Accordingly, the very first access to the variable is a read access (before the variable is written respectively updated in the same computing cycle). Here, the "target REI" is not in the precomputed set of successors.

In other words, no problems occur as long as the producer of data is executed before any consumer. A conflicting scenario would be, e.g., the following: The data produced by "REI-1" is consumed by "REI2", which uses it to produce new data. This data is consumed by "REI-3" which is executed earlier than "REI-1". This results in a conflict due to inconsistency, because "REI-3" uses "old" data for its processing. Therefore the backward dependencies and the cycles formed by them are the core problem. 
AUTOSAR provides several timing constraints that solve such problems. We are using two of them: ExecutionOrderConstraints (EOCs) and AgeConstraints (ACs). The former "[...] is used to specify the order of execution of ExecutableEntities" and the latter "[...] to specify a minimum and maximum age that is tolerated when a variable data prototype is received" (AUTOSAR, 2011).

Referring to the FDs, BDs and AUTOSAR's constraints, our tool starts analyzing and categorizing the identified dependencies. As stated before, it can automatically impose appropriate execution orders for some "straight forward" data dependencies. This is possible if there is a simple constellation like, e.g., two REIs that do not have further dependencies within the system, so that there is no reason to execute the consuming REI first. Hence, a newly created EOC enforces the producing REI to be executed first.

In order to also detect non-trivial conflicts, the analysis continues with subdividing the BDs into "intentional" and "unintentional" ones. The former are characterized by the existence of appropriate ACs explicitly allowing the transfered data to be as old as one computing cycle. If no matching $\mathrm{AC}$ can be found, the BD is probably unintentional. The set of remaining BDs represent critical paths of the system's design that need to be cared for manually by the engineer. Basically, we suggest to explicitly state whether a backward execution order for a data dependency is allowed or not. Permissions for backward execution are represented by ACs whereas EOCs are used for their prevention. By imposing constraints on each potential backward dependency, it is possible to ensure the system's validity with regard to data age consistency.

As a final remark, it is important to mention that cycles can only be formed by data dependencies that actually depend on each other. This means that communication contents (data units) sent to an REI have to be indispensable for the REI's upcoming calculation. This is being considered in our analysis.

\subsection{Plugin and Editing}

The analysis described above is carried out by our tool that visualizes the results and provides semiautomatic problem resolution for the detected conflicting data dependencies.

As stated, EOCs for obvious dependency cases are automatically created. These EOCs are annotated with the text string "computed EOC" in their corresponding AUTOSAR category field. EOCs that are marked like this should not be altered by other tools that use the validated model, e.g., to find a good map- ping of the RE instances to IEUs by means of simulation. Therefore the computed EOCs help to reduce the search space of such "continuative tools".

Handling the remaining conflicts requires direct modification by the software engineer. The tool highlights problematic dependencies with colors and shows necessary resolving actions as tooltips.

By and large, the critical cycles need to be solved by adding ACs and EOCs, removing dispensable EOCs or replacing EOCs with ACs. The specific solution depends on the function and the triggering frequencies of the RE instances involved. One of the displayed hints is, e.g., "Invalid execution order constraint: triggering frequencies do not match!". In this case, another EOC (which would enforce a FD relationship) would not be helpful, so the engineer has to add an AC to resolve the conflict. The new AC denotes the $\mathrm{BD}$ as intentional, which indicates that the use of "old" data (up to one computing cycle) is not a problem. This can be, e.g., the case if the purpose of the corresponding RE instance is monitoring as well as continuously checking certain values' validity. The software engineer does not have to specify the appropriate time value for the AC since it is automatically calculated by the tool.

Depending on the possible selections, the tool shows unproblematic dependencies, allowed BDs, unintentional BDs and imposed EOCs and ACs as colored graphs between the RE instances. The actions applied are directly written to the model which is saved afterwards. The final result is a validated model whose functional blocks are prepared for being distributed to the available execution units.

\section{FINDING ISOLATED REGIONS}

Finding unintentional backward dependencies and solving them with the aid of constraints is a significant step before trying to find a good mapping of "tasks" to multiple IEUs. Having ensured the model's validity, the subsequent search for such a mapping spans a search space that grows exponentially according to the number of RE instances that need to be assigned. Despite the limitations imposed by added constraints, even mid-sized examples (like the braking system introduced in Section 5) involve vast numbers of mapping possibilities.

\subsection{Concept and Definition}

In order to effectively reduce the size of the search space, it is helpful to provide a coarse-grained "prepartition" that assigns as many entities as possible to 
groups. An approach towards this is the search for "entity regions" that are virtually isolated. These regions have only loose coupling with other parts of the system and are therefore promising candidates for the processing on the same Independent Execution Unit.

To extend the analysis of the plug-in, an algorithm inspired by MAF's Single Entry, Single Exit (SESE) analysis is used. This approach bases on (Ottenstein and Ottenstein, 1984; Tip, 1995) as well as (Johnson et al., 1994) and its further development as Token Analysis in (Götz et al., 2009). The goal is to find Single Entry Regions (SERs) within the specified system. Such a region is mainly characterized by the fact that it can only be accessed via one Runnable Entity instance, which is henceforth referred to as "node" due to its graphical illustration by the tool. An "access" encompasses all data dependencies determined by the initial analysis whereas a "path" represents several data dependencies establishing a connection between various nodes. In consequence, a Single Entry Region can be depicted as follows:

- It consists of at least two nodes that are connected by a data dependency.

- The region can only be accessed via one single "entry node".

- Every other node contained in the SER is reachable on a path down starting with the entry node. In other words, every node is either an entry node, a direct or a transitive successor of it.

- Every path ends in a blind end or in one common single exit node (i.e. a final merger node where all dependencies converge).

As it is possible that a Single Entry Region contains entry points besides its initial node, one can infer that several SERs can overlap and even that one SER can be a subset of another.

Looking at the system from a temporal perspective, the data dependencies can be seen as directed graphs between the Runnable Entities (nodes) involved. Hence it is important to take the existence of loops (cycles) into consideration. Following the SER description above, loops within a SER are allowed, because they do not violate its properties. It is worth mentioning that, in contrast to SESE, SER does not mandatorily demand regions to end in one final merger node. For the "isolation property" of a SER, it is not crucial that the dependencies converge in one point. The main issue is the separation of the involved nodes from the rest of the system.

\subsection{Processing and Storing}

The detection of Single Entry Regions requires some pre-computed information about the Runnable Entity instances. Therefore it is helpful to create maps that include - among other things - the nodes' direct successors and direct predecessors (their scope is extended in comparison to the simple "quick access maps" of the pre-analysis).

Using this data, it is easy to determine the parents of the nodes that appear exactly once as direct successor, meaning nodes which are inevitable in order to reach a virtually isolated region. These parent nodes are the Single Entry Points (SEPs) which are used as starting nodes for the SER analysis. The actual determination of the SERs is done by iteratively analyzing the SEPs' successor sets, checking whether the sets' nodes meet the SER conditions and storing discovered isolated regions "behind" a SEP.

Afterwards the collected SERs are filtered to obtain those groups which consist of RE instances having the same period (triggering frequency). S Such groups are particularly suitable for an execution on a common IEU, so that the duration of one "computational iteration" on this IEU is not needlessly delayed due to redundant calculations. If the structural model of the system contains already a mapping of the Software Components (containing the Runnable Entities) to the ECU instances, it is useful to filter the results again in order to obtain SERs that are located on the same ECU. The basic SERs (containing REIs with mixed periods) are not discarded, since they are useful if there is no partition into pieces that contain only REIs with uniform triggering frequencies. Moreover, the proportion between "homogeneous" and "mixed" SERs can hint at the system's suitability for parallelization.

The results of the analysis are written back to the model, which is an AUTOSAR XML file. They are stored as Special Data Groups (SDGs) in AUTOSAR's AdminData, that is located in the root directory of the model right next to the so-called ARPackages which contain the actual description of the system.

The groups are hierarchically structured: the parent nodes SystemView and EcuView each contain SDG collections including Runnable Entity instances of different periods and those with same periods. The listed elements (the RE instances) are represented with their unique path (in the according SpecialData element) and via $S D X$ references pointing directly to the actual element in the system description. The latter eases the further use by another tool.

The Artop plug-in has been extended in order to 
visualize the results of the Single Entry Region analysis. A user can select the desired SER group size to be displayed in a menu list that contains all SER group sizes detected in the analyzed model. The highlighting of the SERs is illustrated in Figure 4.
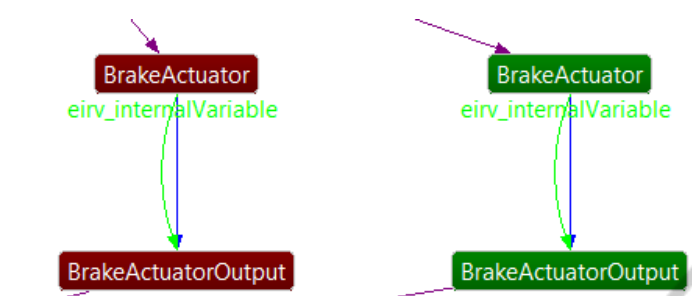

Figure 4: This fitted screen shot shows four nodes (REIs) of the braking system example (see Section 5) as well as the constraints imposed on them (blue EOCs and purple DACs). The nodes' coloring indicates the membership to a specific Single Entry Region.

\section{CASE STUDY}

In order to illustrate the application of our tool, we applied it to the Brake by Wire (BbW) example coming from the TIMMO project respectively its followup project TIMMO-2-USE. TIMMO (TIMing MOdel) "[...] developed a common, standardized infrastructure for the handling of timing information during the design of embedded real-time systems in the automotive industry" (TIMMO, 2007) while the goal of TIMMO-2-USE ("TIMing MOdel - TOols, algorithms, languages, methodology, and USE cases") was to provide tools, algorithms, languages, methodology, and use cases for dealing with timing requirements and properties for timing analyses during the development of distributed embedded automotive systems (TIMMO-2-USE, 2010).

The BbW example was intended to demonstrate the use of the language and methodology for dealing with timing. Applied on different variants of it, the dependency analysis takes about one second when executed by one core of an "Intel i7-3720QM" processor with $2.6 \mathrm{GHz}$. The SER analysis and the graph visualization within the editor do each last less than $100 \mathrm{~ms}$. As the BbW example is a mid-sized model, the duration of the analyses and visualization is quite decent.

Figure 5 shows some RE instances of the BbW example and their status (graph colors) after initially running the analysis. Beginning with this, a software engineer can iteratively fix the problematic dependencies by imposing inevitable constraints. The analysis is automatically rerun after each modification, so that it is easy to track its effects. The final goal is to ob- tain a cycle-free and therefore validated model whose possible execution sequences do not lead to data inconsistencies. Figure 6 partially shows such a model.

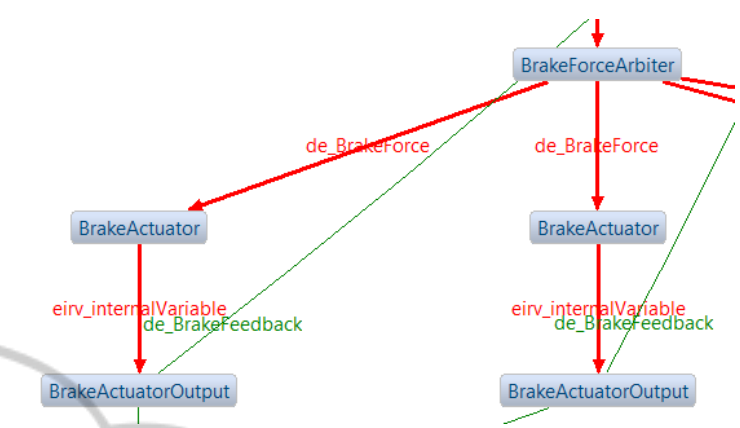

Figure 5: This cropped screenshot shows the results after the first run of the analysis. The dependencies' (graphs') colors indicate the respective dependency type whereas the annotated text shows the affected variables' names. Red lines point to problematic cycles that need to be fixed manually by the software engineer.

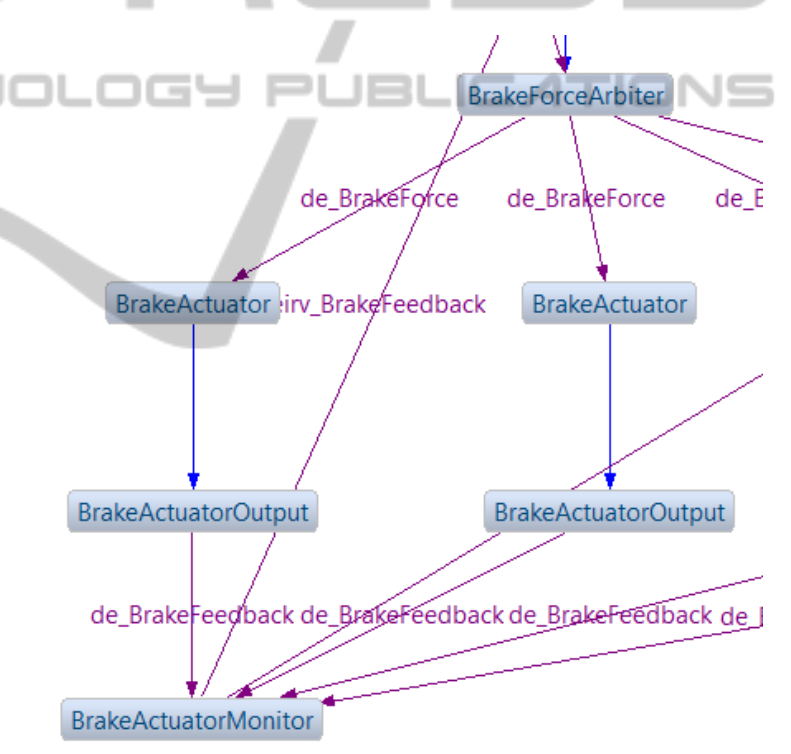

Figure 6: Having validated the model with the help of ACs (purple) and EOCs (blue), the analysis should not find any further problems as shown in this cropped screenshot. The model is now properly prepared for searching a good mapping of RE instances to IEUs.

\section{CONCLUSIONS}

Since the migration to software that runs on multiple IEUs tremendously increases the architectural complexity, our overall goal was to effectively determine ambiguities and inconsistencies in systems modeled with AUTOSAR capabilities and to support their proper resolution.

We achieved this by utilizing existing modeling 
capabilities and combine them with results from research activities, enabling us to immediately make use of it. This is carried out by implementing our Artop-based tool as an Eclipse plug-in that runs its analyses directly on AUTOSAR models and writes back the results and changes performed. More precisely, the tool executes a data dependency analysis on AUTOSAR models, automatically imposes apparent (simple) constraints on the RE instances, highlights existing cycle-related conflicts and supports the software engineer resolving these problems. As the validation of the models ensures that data is produced (written) by an RE instance before it is consumed (read) by another one, the tool continues with the determining suitable sets of virtually isolated RE instances. This is done in order to support a system's parallelization by third party tools that might, e.g., search for an advantageous task to IEU mapping. Thus, other tools do not need to repeat our analysis and are taking advantage of our work.

Our future research will address the significance of dependency analyses as well as the support of parallelization on higher levels of abstraction.

\section{REFERENCES}

Artop Group (2012). AUTOSAR Tool Platform. https://www.artop.org/.(accessed on July 20th, 2013).

AUTOSAR (2011). Specification of Timing Extensions.

AUTOSAR (2013). AUTOSAR Methodology.

Bohn, M., Schneider, J., Eltges, C., and Rößger, R. (2011). Migration von AUTOSAR-basierten Echtzeitanwendungen auf Multicore-Systeme. In Workshop: Entwicklung zuverlässiger Software-Systeme (Stuttgart, Germany).

Deubzer, M., Hobelsberger, M., Mottok, J., Schiller, F., Dumke, R., Siegle, M., Margull, U., Niemetz, M., and Wirrer, G. (2010). Modeling and Simulation of Embedded Real-Time Multicore Systems. In Proceedings of the 3rd Embedded Software Engineering Congress, pages 228-241.

Eclipse Foundation (2009). Eclipse Modeling Framework Project. http://eclipse.org/modeling/emf/, accessed on July 15th, 2013).

Eißenlöffel, T. (2012). Embedded-Software entwickeln. dpunkt.

Gehrke, M., Nawratil, P., Niggemann, O., Schäfer, W., and Hirsch, M. (2006). Scenario-based verification of automotive software systems. In MBEES, pages 35-42.

Gleim, U. and Schüle, T. (2012). Multicore-Software. dpunkt.

Götz, M., Roser, S., Lautenbacher, F., and Bauer, B. (2009). Token analysis of graph-oriented process models. In Enterprise Distributed Object Computing Conference Workshops, 2009. EDOCW 2009. 13th, pages 15-24.
Johnson, R., Pearson, D., and Pingali, K. (1994). The program structure tree: Computing control regions in linear time. In ACM SigPlan Notices, volume 29, pages 171-185. ACM.

Multicore, A. (2011). Relevanz eines MulticoreÖkosystems für künftige Embedded Systems: Positionspapier zur Bedeutung, Bestandsaufnahme und Potentialermittlung der Multicore-Technologie für den Industrie-und Forschungsstandort Deutschland.

Ottenstein, K. J. and Ottenstein, L. M. (1984). The Program Dependence Graph in a Software Development Environment. In ACM Sigplan Notices, volume 19, pages 177-184.

Padberg, F. and Denninger, O. (2013). MulticoreSoftwarefehler im Visier: Automatische Fehlererkennung in Entwürfen paralleler Programme. OBJEKTspektrum, Ausgabe 01/2013, 20(1):72-76.

Patterson, D. (2010). The trouble with multi-core. Spectrum, IEEE, 47(7):28-32.

Saad, C. (2009). Model Analysis Framework. http:// www.informatik.uni-augsburg.de/en/chairs/ $\mathrm{swt} / \mathrm{ds} /$ projects/mde/maf/. (accessed on July 20th, 2013).

Saad, C. and Bauer, B. (2013).- Data-flow based Model Analysis and its Applications. (accepted for ACM/IEEE 16th International Conference on Model Driven Engineering Languages and Systems, 29 September 2013 through 4 October 2013, Miami, USA).

Schäuffele, J. and Zurawka, T. (2010). Automotive Software Engineering. Springer DE.

Shih, C., Wu, C.-T., Lin, C.-Y., Hsiung, P.-A., Hsueh, N.-L., Chang, C.-H., Koong, C.-S., and Chu, W. C. (2009). A model-driven multicore software development environment for embedded system. In Computer Software and Applications Conference, 2009. COMPSAC'09. 33rd Annual IEEE International, volume 2, pages 261-268. IEEE.

Sodan, A. C., Machina, J., Deshmeh, A., Macnaughton, K., and Esbaugh, B. (2010). Parallelism via multithreaded and multicore CPUs. Computer, 43(3):24-32.

Sutter, H. (2005). The Free Lunch Is Over: A Fundamental Turn Toward Concurrency in Software. Dr. Dobbs Journal, 30(3):202-210.

TIMMO (2007). Timing Model. http:// www.itea2.org/project/index/view?project=170. (accessed on July 18th, 2013).

TIMMO-2-USE (2010). TIMMO Braking System. http:// www.timmo-2-use.org/. (accessed on July 18th, 2013).

Tip, F. (1995). A Survey of Program Slicing Techniques. Journal of programming languages, 3(3):121-189.

Wirbel, L. (2011). Embedded Multicore Goes Mainstream. http://www.designnews.com/author.asp?section_id= 1386\&doc_id=231676. (accessed on July 15th, 2013). 